\title{
Educação física escolar e obesidade em escolares portadores de Síndrome de Down
}

\author{
Tácio Rodrigues da Silva Santos ${ }^{1}$ \\ Francisco Martins da Silva ${ }^{2}$ \\ Renata Aparecida Elias Dantas ${ }^{3}$ \\ Sérgio Adriano Gomes ${ }^{4}$ \\ Marcelo Guimarães Bóia do Nascimento ${ }^{5}$ \\ Márcio Rabelo Mota ${ }^{6}$
}

\section{Resumo}

A literatura científica relata níveis de obesidade extremamente elevados entre a população de portadores de Síndrome de Down, bem como relata a intervenção por meio de exercício físico como um dos pilares básicos do tratamento da obesidade. A Educação Física como uma prática pedagógica ligada aos esportes, aos jogos, às lutas, às ginásticas e às danças dentro das instituições de ensino demonstra ser um agente capaz de desempenhar o papel do exercício físico no tratamento da obesidade em escolares portadores de Síndrome de Down; além de proporcionar benefícios que são de grande valia para dimensão psicológica e social do ser humano.

Palavras-chave: Cromossomopatia. Trissomia 21. Hipernutrição. Escola.

\section{Introdução}

A obesidade é um estado de composição corporal no qual alterações fisiológicas, anatômicas e psicológicas interagem como causa e efeito do acúmulo de

\footnotetext{
Discente do curso de Educação Física do UniCEUB; e-mail: tacio.rss@gmail.com Docente do curso de Educação Física da UCB; e-mail: fmsilva@ucb.br

Docente do curso de Educação Física do UniCEUB; e-mail: profrenataelias@yahoo.com.br

4 Docente do curso de Educação Física do UniCEUB; e-mail: adrianofutsal@yahoo.com.br

Docente do curso de Educação Física do UniCEUB; e-mail: marcelo.boia@gmail.com

6 Docente do curso de Educação Física do UniCEUB; e-mail: marciormota@gmail.com
} 
gordura no organismo em níveis prejudiciais à saúde (ANGELIS, 2003; SOARES; PETROSKI, 2003; WORLD HEALTH ORGANIZATION, 2000).

O número de casos de obesidade no Brasil avança entre indivíduos de todas as idades e em todos os níveis socioeconômicos. Esse aumento é mais expressivo entre as mulheres de famílias de baixa renda (FERREIRA; MAGALHÃES, 2006). De acordo com Silva (2010), a obesidade atinge 37,5\% da população brasileira.

A Síndrome de Down (SD) é a cromossomopatia mais comum da espécie humana, sendo uma das poucas em que existe a possibilidade de não haver aborto espontâneo. As especificidades que caracterizam tal síndrome acontecem em função de uma falha no processo de divisão celular da formação fetal, conhecido como não-disjunção, no qual um cromossomo sobressalente se une ao par de cromossomos número 21 nas células do organismo, constituindo uma situação classificada como trissomia 21 (CARNEIRO, 2008; KORZMA, 2007; PUESCHEL, 2007a).

Existem aproximadamente 300 mil portadores de SD no Brasil. A patologia é a décima sexta causa de morte reduzível por ações adequadas de diagnóstico e tratamento do Sistema Único de Saúde (SUS), entre menores de 5 anos de idade, nascidos em território nacional (IBGE, 2002; MALTA et al., 2007).

A prevalência de obesidade entre portadores de SD é extremamente elevada quando observada em comparação com os números descritos entre indivíduos típicos. Existem pesquisas que apontam uma incidência superior a $50 \%$ da população estudada (MENDONÇA; PEREIRA, 2008; SILVA, 2010; SILVA et al., 2006).

A principal estratégia no tratamento da obesidade é o ajuste do balanço energético composto pela relação entre a ingestão proporcionada pela dieta e o gasto exigido do organismo para desempenhar a rotina imposta a ele (ANGELIS, 2003; AVESANI et al., 2005; HAUSER et al., 2004; WORLD HEALTH ORGANIZATION, 2000). No caso do portador de SD, quando esse ajuste acontece pelo incremento do gasto energético por meio do exercício físico, observam-se também melhoras funcionais que tornam o indivíduo menos dependente de cuidados alheios (MARQUES; NAHAS, 2003). 
A Educação Física como disciplina da grade curricular regular tem como objetivo proporcionar uma formação integral e culturalmente crítica ao educando. Com esse foco, e de acordo com a sua origem tradicionalmente ligada ao exercício físico, essa disciplina deve ser capaz de proporcionar informação, autonomia e consciência corporal suficiente para a adoção voluntária de um estilo de vida fisicamente ativo (BRASIL, 1997; GUEDES, 1999; BETTI; ZULIANI, 2002). Nessa conjuntura, a Educação Física escolar demonstra ser um agente de intervenção expressiva no tratamento da obesidade entre portadores de SD em idade escolar (SILVA et al., 2008b).

De acordo com o contexto apresentado, este trabalho consiste em um estudo descritivo de revisão bibliográfica, com o objetivo de demonstrar a intervenção da Educação Física escolar no tratamento da obesidade em escolares portadores de SD.

\section{Obesidade}

Todas as atividades voluntárias e involuntárias desempenhadas pelo organismo humano dependem de alguma quantidade de energia para serem desempenhadas. A dieta deve fornecer um aporte energético suficiente, para que as atividades necessárias ao indivíduo que a ingere, sejam possíveis (MAUGHAN; BURKE, 2004).

O peso corporal e a composição corporal são resultado de um balanço energético entre a energia ingerida e a energia gasta. Esse balanço é negativo quando a ingestão é menor que o gasto (resultando em perda de peso), neutro, quando a ingestão é igual ao gasto (resultando em manutenção de peso), e positivo, quando a ingestão é maior que o gasto (resultando em ganho de peso). Nos casos de balanço energético positivo, a energia excedente tende a ser armazenada pelo organismo sob a forma de moléculas de gordura, primordialmente concentradas no tecido adiposo, aumentando o volume dessa estrutura (ANGELIS, 2003; AVESANI et al., 2005).

Estima-se que $40 \%$ a $70 \%$ das alterações de fenótipo, características da obesidade, se devam à pré-disposição genética. Essas alterações são identificadas frequentemente em situações de distúrbio alimentar e/ou alterações metabólicas que comprometem o gasto energético (MARQUES-LOPEZ et al., 2004). 
Existem cinco mutações genéticas observadas em modelos animais que estão relacionadas ao fenótipo obeso: yelow $\left(A^{4}\right)$ no cromossomo 2, diabético $(d b)$ no cromossomo 4, obeso ( $o b)$ no cromossomo 6, tubby $(t u b)$ no cromossomo 7 e fatty (fat) no cromossomo 8 (DAMIANI, 2007).

O $o b$ se expressa apenas no tecido adiposo. É o gene mais abordado nas pesquisas que estudam a genética da obesidade em função da sua capacidade de codificar a leptina - hormônio proteico secretado pelos adipócitos, que age no hipotálamo de modo a regular as sensações de apetite e saciedade (ANGELIS, 2003; DAMIANI, 2007; MARQUES-LOPES et al., 2004; VIUNISK, 1999). O volume elevado de tecido adiposo presente no organismo do indivíduo obeso promove uma secreção elevada de leptina, que faz com que a grande concentração plasmática do hormônio torne o organismo resistente ao efeito anorético desse peptídeo, normalmente interpretado como sensação de saciedade (ANGELIS, 2003; DAMIANI, 2007).

É possível visualizar que a pré-disposição genética é um dos principais fatores de risco para a ocorrência da obesidade, em um estudo realizado por Sorensen et al. (1989). Nessa casuística, foi possível obter dados sobre o IMC de 3.580 indivíduos criados por famílias adotivas, bem como sobre o IMC dos pais adotivos e dos pais biológicos desses indivíduos. Os sujeitos foram divididos em quatro níveis de massa corporal, independente da relação entre eles: magro, peso médio, acima do peso e obeso. Após essa divisão, buscou-se verificar se havia alguma relação entre o enquadramento dos filhos e de seus pais adotivos e entre o enquadramento dos filhos e de seus pais biológicos. Não foi possível identificar nenhuma relação estatisticamente significativa entre a classificação dos filhos e de seus pais adotivos, ao passo que houve uma relação de alta consideração estatística entre a classificação dos filhos e de seus pais biológicos.

O outro principal fator de risco para a ocorrência da obesidade é a prédisposição ambiental, em que o sedentarismo e a incorporação de alimentos inadequados à dieta, que, ao contrário da impressão mantida no senso comum, não acontece mais apenas entre famílias de maior poder aquisitivo - contribuem para formatar um balanço energético positivo capaz de conduzir o indivíduo à obesidade, até mesmo em casos em que não há pré-disposição genética 
(FONSECA et al., 1998; MONDINI; MONTEIRO, 1998; SAUER et al., 2009; SOARES; PETROSKI, 2003).

\section{Síndrome de Down}

As alterações fenotípicas do portador de SD podem ser visíveis desde o estágio fetal, por meio de ultrassonografia, e são facilmente perceptíveis no momento do parto. A presença de características como olhos em formato oriental, nariz pequeno, orelha abaixo da posição natural, língua protusa, pescoço encurtado, pelve de tamanho reduzido, distância entre o hálux e o segundo pododáctilo maior que o padrão e extremidades do corpo com aparência encurtada e volumosa, quando observadas em conjunto, sugerem tratar-se de um caso de SD. Nessa condição, a confirmação ou negação da suspeita é realizada por meio da análise dos cromossomos de uma amostra tecidual por meio de cariograma ${ }^{7}$. Quando o diagnóstico é positivo, o cariograma permite também identificar o tipo de trissomia que ocorre (Tabela 1) (CARNEIRO, 2008; MORENO, 1996).

A SD gera as seguintes malformações e enfermidades (KOIFFMAN et al., 1996):

- Olhos:afastamento excessivo da cavidade orbicular(Hipertelorismo Ocular), manchas esbranquiçadas nas íris (Manchas de Brushfield), inflamações nas pálpebras (Blefarite), esforço maior de um olho que do outro (Estrabismo Não-paralítico) e opacidade do globo ocular (Catarata Senil);

- Ouvidos: canal auditivo estreito, com os ossos do ouvido médio malformados, causando perda da condução auditiva ao cérebro (Surdez Neutrossensorial);

- Nariz: narinas antevertidas, de base plana, com uma hipoplasia ${ }^{8}$ óssea;

7 Exame que consiste na ilustração gráfica individualizada dos cromossomos de uma ou mais células.

8 Redução do volume tecidual em função de uma quantidade inferior de células no tecido em questão. 
- Boca: palato em formato ogival, dentes malformados e língua extensa - que com o avanço da idade tende a se tornar fissurada;

- Mão: polegar curvado em direção medial (Clinodactilia) e hipoplasia na falange média dos dedos;

- Abdome: separação entre a face direita e esquerda do músculo reto abdominal (Diástase) e hérnia umbilical; e

- Coluna: hiperflexibilidade da articulação atlanto-axial e estreitamento do canal cervical.

Uma série de possíveis causas da não-disjunção foi investigada no decorrer dos anos, tais como a exposição ao Raio-x, a administração de determinadas drogas, alterações hormonais, alterações imunológicas e espermatocidas; mas a única característica que demonstra relação comprovada com esse erro de divisão cromossômica é a idade materna avançada (KOZMA, 2007; PUESCHEL, 2007b).

A probabilidade de ocorrência de SD aumenta de acordo com o avanço da idade materna durante a gestação e se torna consideravelmente expressiva após os 35 anos de idade (Tabela 2). O fato de 75\% dos casos de SD acontecer em gestações em que a idade materna é inferior a esta acontece em razão de uma quantidade maior de mulheres engravidarem com menos de 35 anos, e não invalida tal projeção (KOZMA, 2007).

Tabela 1: Tipos de Síndrome de Down.

\begin{tabular}{|c|c|c|}
\hline Tipo & Característica & Prevalência \\
\hline Trissomia 21 & $\begin{array}{l}\text { Quando todas as células do organismo } \\
\text { possuem } 47 \text { cromossomos. }\end{array}$ & $\begin{array}{l}\text { Cerca de } 95 \% \text { dos } \\
\text { casos }\end{array}$ \\
\hline Translocação & $\begin{array}{l}\text { Quando o cromossomo extra ligado } \\
\text { ao vigésimo primeiro par faz parte de } \\
\text { outro par de cromossomos (geralmente } \\
\text { o décimo quarto ou o vigésimo } \\
\text { segundo). }\end{array}$ & $\begin{array}{l}\text { Cerca de } 3 \% \text { a } 4 \% \\
\text { dos casos }\end{array}$ \\
\hline Mosaicismo & $\begin{array}{l}\text { Quando a não-disjunção atinge apenas } \\
\text { parte das células, fazendo com que } \\
\text { algumas delas tenham } 47 \text { cromossomos } \\
\text { e outras } 46 .\end{array}$ & $\begin{array}{l}\text { Cerca de } 1 \% \text { dos } \\
\text { casos }\end{array}$ \\
\hline
\end{tabular}

Fonte: PUESCHEL (2007a). 
Tabela 2: Probabilidade de ocorrência de Síndrome de Down de acordo com a idade materna durante a gestação.

\begin{tabular}{|c|c|c|c|c|c|}
\hline $\begin{array}{c}\text { Idade } \\
\text { Materna }\end{array}$ & $\begin{array}{c}\text { Risco } \\
\text { Estimado }\end{array}$ & $\begin{array}{c}\text { Idade } \\
\text { Materna }\end{array}$ & $\begin{array}{c}\text { Risco } \\
\text { Estimado }\end{array}$ & $\begin{array}{c}\text { Idade } \\
\text { Materna }\end{array}$ & $\begin{array}{c}\text { Risco } \\
\text { Estimado }\end{array}$ \\
\hline 20 & $1: 1231$ & 30 & $1: 585$ & 40 & $1: 78$ \\
\hline 21 & $1: 1145$ & 31 & $1: 530$ & 41 & $1: 51$ \\
\hline 22 & $1: 1065$ & 32 & $1: 563$ & 42 & $1: 47$ \\
\hline 23 & $1: 1000$ & 33 & $1: 452$ & 43 & $1: 37$ \\
\hline 24 & $1: 942$ & 34 & $1: 352$ & 44 & $1: 20$ \\
\hline 25 & $1: 887$ & 35 & $1: 271$ & 45 & $1: 22$ \\
\hline 26 & $1: 842$ & 36 & $1: 213$ & 46 & $1: 17$ \\
\hline 27 & $1: 798$ & 37 & $1: 155$ & 47 & $1: 13$ \\
\hline 28 & $1: 755$ & 38 & $1: 129$ & 48 & $1: 10$ \\
\hline 29 & $1: 721$ & 39 & $1: 100$ & 49 & $1: 8$ \\
\hline
\end{tabular}

Fonte: KOZMA (2007)

Beiguelman et al. (1996) e Gusmão et al. (2003) realizaram dois estudos semelhantes, ilustraram, respectivamente, a relação entre a idade materna durante a gestação e a ocorrência de SD nas regiões Sudeste e Nordeste do Brasil. Ambos os autores descrevem um aumento da ocorrência de SD atrelado ao avanço da idade materna até aproximadamente os 30 anos de idade, quando a quantidade de casos reduz em função de um decréscimo na quantidade total de nascimentos.

\section{Obesidade na Síndrome de Down}

A pré-disposição genética à obesidade se expressa na SD por meio, sobretudo, das alterações anatomo-fisiológicas que impõem ao portador dessa cromossomopatia um consumo de oxigênio mais elevado que o de indivíduos típicos. Os níveis de ventilação do portador de SD exigem que a mobilização de moléculas de gordura, como principal fonte de energia durante o repouso, seja substituída pela mobilização de moléculas de carboidrato. Essa alteração do macronutriente predominantemente empregado como combustível para as reações metabólicas favorece expressivamente o acúmulo de moléculas de gordura no tecido adiposo (SILVA et al., 2007). 
A pré-disposição ambiental do portador de SD à obesidade pode ser notada na frequente desadequação dietética observada entre os indivíduos que apresentam a cromossomopatia. Quase 60\% dos portadores de SD apresentam hábitos alimentares inadequados, sendo que em $83 \%$ dos casos nos quais a alimentação não segue o padrão recomendado, percebe-se que, durante a infância, os pais não receberam orientações sobre as especificidades que a alimentação dos seus filhos deveria seguir (MINGRONI, 1997; SIMÕES et al., 2007).

Diferentes tipos de suplementação alimentar já foram administradas a fim de contrabalançar as situações que favorecem o acúmulo de moléculas de gordura no tecido adiposo em portadores de SD, mas nenhuma das intervenções descritas com tais objetivos apresentou resultados significativos a ponto de justificar seu emprego (ELLIS et al., 2008, ROMANO et al., 2002).

\section{Educação física escolar e obesidade na Síndrome de Down}

As primeiras escolas a admitir educandos portadores de SD eram instituições de educação especial, que, normalmente, funcionavam em regime de internato. Posteriormente, esses educandos começaram a ser inseridos em classes especiais de escolas regulares (GONZÁLES, 2007).

O desafio atual da educação de educandos portadores de SD é proporcionar-lhes a aquisição dos conhecimentos necessários para a inclusão no ensino regular. A dificuldade em alcançar esse objetivo não se deve apenas ao fato de o desenvolvimento neuropsicomotor na SD ser diferente do padrão referencial, mas também à falta de ações pedagógicas que desenvolvam as habilidades sociais desses indivíduos de maneira sistemática e produtiva (BISSOTO, 2005; GONZÁLEZ, 2007, SANTIS et al., 2004; ZAUSMER, 2007).

A formação educacional do educando portador de SD não deve se resumir apenas a um contexto escolar e/ou terapêutico que busca entender seu desenvolvimento como uma versão mais lenta do desenvolvimento típico (BISSOTO, 2005; VOIVODIC; STORER, 2003). O processo de ensino-aprendizagem nesses casos 
deve ser planejado levando em consideração que não existe um padrão nas diferenças entre o desenvolvimento do portador de SD e do indivíduo típico, pois mesmo com a cromossomopatia, o portador de SD possui 22 pares de cromossomos saudáveis que lhe conferem a sua individualidade biológica (JACKSON-COOK, 1996; MANCINI, 2003).

A Educação Física escolar proporciona possibilidades pedagógicas irrestritas quando o movimento não é encarado como um fim, e sim como um meio de familiarização do educando com o seu próprio corpo. Sob essa perspectiva, é possível conduzir o educando portador de SD a qualquer objetivo, sem que existam impedimentos causados pelas limitações do seu desenvolvimento motor (SILVA; FERREIRA, 2001).

Um programa de Educação Física escolar deve promover a maior variedade de experiências possíveis ao educando portador de SD, pois cada tipo de estímulo gera adaptações que tornam mais fácil experimentar situações que guardam alguma semelhança com o que já foi vivenciado. Desse modo, é possível promover intervenções que não se restringem apenas ao aprimoramento do desenvolvimento motor, auxiliando também no processo de desenvolvimento cognitivo (ORNELAS; SOUZA, 2001). Em casos em que esas características se aplicam, observa-se que os educandos portadores de SD apresentam um comportamento mais ameno, com mais autoestima e mais autoconfiança, além de uma vontade constante de buscar desafios e sensações ligados ao sentimento de autossuperação (SILVA et al. 2008a; SILVA et al., 2008b).

O sentimento de autossuperação proporciona ao educando portador de SD uma motivação que permite considerar o emprego de qualquer atividade compreendida como conteúdo da Educação Física, usualmente empregado no tratamento da obesidade entre indivíduos típicos (BRASIL, 1997; GORDON, 2006; MILLEN NETO; SILVA et al., 2008a; SILVA et al., 2008b).

É possível observar que tanto o exercício físico aeróbio (EA) como o exercício físico resistido (ER) promovem alterações favoráveis na composição corporal do portador de SD, logo, esses dois tipos de exercício físico podem ser empregados na Educação Física escolar com o objetivo de tratar a obesidade (SANTOS, 2008). 
Um estudo realizado por Carmeli et al. (2004) demonstrou que 13 semanas de EA em intensidade autossugerida são suficientes para promover um aumento significativo na performance aeróbia de portadores de SD. As avaliações pré e pós-intervenção demonstraram aumento de $193 \%$ na distância percorrida, $88 \%$ na velocidade alcançada e $156 \%$ na duração do exercício físico. Esses resultados foram alcançados com sessões de no máximo 40 minutos, realizadas em três dias alternados por semana e demonstram que o EA condiciona portadores de SD a realizar esforços cada vez mais intensos - que, consequentemente, promovem cada vez mais gasto energético.

Florentino Neto et al. (2009) realizaram um estudo cujos resultados descrevem que 12 semanas de ER em forma de circuito podem promover uma redução significativa da quantidade de gordura corporal subcutânea em portadores de SD (2\%). Esse resultado foi alcançado com sessões de 60 minutos em três dias alternados na semana. As sessões consistiam em movimentos que priorizavam os grandes grupamentos musculares, com a execução de três séries compostas por 8 a 12 repetições em cada exercício, separadas por intervalos de 30 a 60 segundos.

O EA e o ER são agentes extremamente eficazes no tratamento da obesidade em portadores de SD, mas os programas de Educação Física escolar devem explorar tais elementos de uma maneira que não cause a esportivização excessiva da prática pedagógica. O objetivo fisiológico da intervenção deve estar camuflado entre atividades aparentemente lúdicas, simples e atrativas (BRASIL, 1997; CARMELI et al., 2004; GORDON, 2006; FLORENTINO NETO et al., 2009; SANTOS, 2008).

\section{Considerações finais}

As alterações anatomo-fisiológicas geradas pela presença do cromossomo extra no núcleo celular que caracteriza a SD maximizam os principais fatores de risco para a ocorrência da obesidade - a pré-disposição genética e a pré-disposição ambiental.

A intervenção da Educação Física escolar não é capaz de anular esses fatores de risco para a ocorrência de obesidade maximizada pela SD, mas pode atenuá-los, aumentando o gasto energético para formatar um balanço energético negativo. 
Em anexo ao efeito fisiológico descrito, a Educação Física escolar também proporciona benefícios ao educando portador de SD que são de grande valia para dimensão psicológica e social do ser humano; como autoestima, autoconfiança, desejo de autossuperação e maior facilidade com as questões relacionadas às relações interpessoais.

\title{
School physical education and obesity in Down Syndrome students
}

\begin{abstract}
The scientific literature describes highly elevate obesity levels among Down's syndrome population, insofar physical exercise's intervention is described like a base pillar on the obesity treatment. Scholar Physical Education as a pedagogic practice related to sports, games, martial arts, dance and gymnastics inside the educational institutions shows to be capable of execute physical exercise's intervention function on the scholar Down's syndrome individuals obesity treatment; furthermore also to promote great psychological and social human dimension benefits.
\end{abstract}

Keywords: Chromosomopathy. Trisomy 21. Hypernutrition. School.

\section{Referências}

ANGELIS, R. C. Riscos e prevenção da obesidade: fundamentos fisiológicos e nutricionais para tratamento. São Paulo: Atheneu, 2003.

AVESANI, C. M. et al. Necessidades e recomendações de energia. In: CUPPARI, L. Guia de nutrição: nutrição clínica no adulto. Barueri: Manole, 2005.

BEIGUELMAN, B. et al. Maternal age and Down syndrome in Southeastern Brazil. Revista Brasileira de Genética, [S.1], v. 19, n. 4, p. 367-640, 1996. 
BETTI, M.; ZULIANI, L. R. Educação física escolar: uma proposta de diretrizes pedagógicas. Revista Mackenzie de Educação Física e Esporte, São Paulo, v. 1, n. 1, p. 73-81, 2002.

BISSOTO, M. L. Desenvolvimento cognitivo e o processo de aprendizagem do portador de Síndrome de Down: revendo concepções e perspectivas educacionais. Ciências \& Cognição, [S.1], v. 4, p. 80-88, 2005.

BRASIL. Secretaria de Educação Fundamental. Parâmetros curriculares nacionais: educação física. Brasília: MEC/SEF, 1998.

CARMELI, E. et al. Impact of a walking program in people with Down Syndrome. Journal of Strength and Conditioning Research, [S.1], v. 18, n. 1, p. 180-184, 2004.

CARNEIRO. M. C. Adultos com Síndrome de Down. Porto Alegre: Artmed, 2008.

DAMIANI, D. Tecido adiposo como órgão endócrino. In: SETIAN, N. et al. Obesidade na criança e no adolescente: buscando caminhos desde o nascimento. São Paulo: Roca, 2007. p. 29-44.

ELLIS, J. M. et al. Supplementation with antioxidants and folinic acid for children with Down's Syndrome: randomised controlled trial. British Medical Journal, [S.l], v. 336, p. 594-597, 2008.

FERREIRA, V. A.; MAGALHÃES, R. Obesidade no Brasil: tendências atuais. Revista Portuguesa de Saúde Pública, [S.l], v. 24, n. 2, p. 71-81, 2006.

FLORENTINO NETO, J. et al. Impacto de 12 semanas de treinamento de força sobre a composição corporal de portadores de síndrome de Down. Revista da AMRIGS, [S.1], v. 53, n. 1, p. 11-15, 2009.

FONSECA, V. M. et al. Fatores associados à obesidade em adolescentes. Revista de Saúde Pública, [S.1], v. 32, n. 6, p. 541-549, 1998.

GONZÁLEZ, E. Necessidades educacionais específicas. Porto Alegre: Artmed, 2007.

GORDON, D. The benefits of exercise to a Down's syndrome population. 2006. Disponível em: <http://www.intellectualdisability.info/physical-health/thebenefits-of-exercise-to-a-downs-syndrome-population>. Acesso em: 2 jun. 2009.

GUEDES, D. P. Educação para a saúde mediante programas de educação física escolar. Motriz, [S.l], v. 5, n. 1, p. 10-14, 1999. 
GUSMÃO, F. F. et al. Idade materna e síndrome de Down no Nordeste do Brasil. Caderno de Saúde Pública, [S.1], v. 19, n. 3, p. 973-978, 2003.

HAUSER, C. et al. Estratégias para o emagrecimento. Revista Brasileira de Cineantropometria \& Desempenho Humano, [S.1], v. 6, n. 1, p. 72-81, 2004.

INSTITUTO BRASILEIRO DE GEOGRAFIA E ESTATÍSTICA. Censo 2000. Brasília, 2003. Disponível em: <http://www.ibge.gov.br>. Acesso em: 16 mar. 2009.

KOIFFMAN, C.P. et al. Cromossomopatias. In: DIAMENT, A. CYPEL, S. Neurologia infantil. São Paulo: Atheneu, 1996. p. 305-335.

KOZMA, C. O que é Síndrome de Down. In: STRAY-GUNDERSEN, K. Crianças com síndrome de Down: guia para pais e educadores. Porto Alegre: Artmed, 2007. p. $14-42$.

MALTA, D. et al. Lista de causas de mortes evitáveis por intervenções do Sistema Único de Saúde do Brasil. Epidemiologia e Serviço de Saúde, [S.1], v. 16, n. 2, p. 233-243, 2007.

MANCINI, M.C. et al. Comparação do desempenho funcional de crianças portadoras de síndrome de Down e crianças com desenvolvimento normal aos 2 e 5 anos de idade. Arquivos de Neuropsiquiatria, [S.1], v. 61, n. 2-B, p. 409-415, 2003.

MARQUES, A. C. NAHAS, M. V. Qualidade de vida de pessoas portadoras de Síndrome de Down com mais de 40 anos no estado de Santa Catarina. Revista Brasileira de Ciência e Movimento, [S.1], v. 11, n. 2, p. 55-61, 2003.

MARQUES-LOPES, I. et al. Aspectos genéticos da obesidade. Revista Brasileira de Nutrição, [S.1], v. 17, n. 3, p. 327-338, 2004.

MAUGHAN, R. J.; BURKE, L. M. Nutrição esportiva. Porto Alegre: Artmed, 2004.

MENDONÇA, G. PEREIRA, F. Medidas de composição corporal em adultos portadores de Síndrome de Down. Revista Brasileira de Educação Física e Esporte, [S.1], v. 22, n. 3, p. 201-210, 2008.

MILLEN NETO, A. M.; SILVA, T. C. Inclusão educacional de alunos com síndrome de Down. Pensar a Prática, [S.1], v. 11, n. 2, p. 105-113, 2008.

MINGRONI, D. P. Alimentação na síndrome de Down: relação pais-criança. 1997. 25 f. Monografia (Especialização)-CEFAC, São Paulo, 1997. 
MONDINI, L. MONTEIRO, C. A. Relevância epidemiológica da desnutrição e da obesidade em distintas classes sociais: métodos de estudo e aplicação à população brasileira. Revista Brasileira de Epidemiologia, [S.1], v. 1, n. 1, p. 28-39, 1998.

MORENO, G. Síndrome de Down: um problema maravilhoso. Brasília: Corde, 1996.

ORNELAS, M. A. SOUZA, C. A contribuição do profissional de educação física na estimulação essencial em crianças com síndrome de Down. Revista de Educação Física/UEM, [S.1], v. 12, n. 1, p. 77-88, 2001.

PUESCHEL, S. M. Panorama histórico. In: PUESCHEL, S. M. Síndrome de Down: guia para pais e educadores. Campinas: Papirus, 2007a. p. 45-51.

PUESCHEL, S. M. Causas da síndrome de Down. In: PUESCHEL, S. M. Síndrome de Down: guia para pais e educadores. Campinas: Papirus, 2007b. p. 53-63.

ROMANO, C. et al. Is there a relationship between zinc and the peculiar comorbidities of Down syndrome? Down Syndrome Research and Practice, [S.l], v. 8 , n. 1, p. 25-28, 2002.

SANTIS, F. A. et al. Desarrollo de habilidades sociales: un camino a la integración social de los niños con síndrome de Down del colegio especial Alameda de La Comuna de estación central. 2004. 134 f. Monografia (Graduação)-Universidad Santo Tomás de Santiago, Santiago, 2004.

SAUER, P. et al. Perfil alimentar de pacientes com sobrepeso e obesidade atendidos em uma clínica de Porto Alegre entre os anos de 2000 e 2006. In: SALÃO DE INICIAÇÃO CIENTÍFICA - PUCRS, 9., 2009, Porto Alegre. . Resumos... Porto Alegre: PUCRS, 2009, p. 881.

SANTOS, N. P. P. Análise da influência da estimulação musical no decurso de uma prova de resistência aeróbia em indivíduos com Síndrome de Down. 2008. 182 f. Dissertação (Mestrado)-Universidade do Porto, Porto, 2008.

SILVA, D. L. et al. Avaliação da composição corporal em adultos com Síndrome de Down. Arquivos de Medicina, [S.1], v. 20, n. 4, p. 103-110, 2006.

SILVA, D. S. et al. A importância da coordenação motora na ginástica rítmica desportiva com portadores com síndrome de Down. In: CONGRESSO CIENTÍFICO NORTE-NORDESTE - CONAFF, 5., 2008, Fortaleza. Livro de Memórias do $5^{\circ}$ Congresso Científico Norte-nordeste. Fortaleza: CONAFF, 2008a, p. 30. 
SILVA, D. R.; FERREIRA, J. S. Intervenções na educação física em crianças com síndrome de Down. Revista de Educação Física/UEM, [S.1], v. 12, n. 1, p. 69-76, 2001.

SILVA, I. M. et al. Percepção dos portadores de síndrome de Down da APAE sobre os benefícios proporcionados pelas aulas de educação física na cidade de Juazeiro do Norte/CE. In: CONGRESSO CIENTÍFICO NORTE-NORDESTE - CONAFF, 5., 2008b, Fortaleza, CE. Livro de Memórias do 5 Congresso Científico Nortenordeste. Fortaleza: CONAFF, 2008, p. 20.

SILVA, M. B. et al. Análise do consumo de oxigênio em portadores da síndrome de Down. In: ENCONTRO LATINO AMERICANO DE INICIAÇÃO CIENTÍFICA E ENCONTRO LATINO AMERICANO DE PÓS GRADUAÇÃO, 11 e 7., 2007, São José dos Campos. Anais... São José dos Campos, 2007.

SILVA, V. S. Prevalência de sobrepeso/obesidade e fatores associados em adultos no Brasil 2010. 78 f. Dissertação (Mestrado)-Universidade Federal de Santa Catarina, Santa Catarina, 2010.

SIMÕES, L. C. et al. Avaliação nutricional e antropométrica de crianças de 06 a 36 meses, com síndrome de Down, e oferta de oficinas de culinária à suas famílias - Fundação Dom Bosco - B.H. In: SEPEX - SEMANA DE PESQUISA E EXTENSÃO DO UNIBH - UNIBH, 4., 2007, Belo Horizonte. Resumos... Belo Horizonte: UniBH, 2007. p. 1.

SOARES, L. D. PETROSKI, E.L. Prevalência, fatores etiológicos e tratamento da obesidade infantil. Revista Brasileira de Cineantropometria \& Desempenho Humano, [S.1], v. 5, n. 1, p. 63-74, 2003.

SØRENSEN, T. A. Genetics of obesity in adult adoptees and their biological siblings. British Medicine Journal, [S.1], v. 298, p. 87-90, 1989.

VIUNISK, N. Obesidade Infantil: um guia prático para profissionais de saúde. São Paulo: Publicações Biomédicas, 1999.

VOIVODIC, M. A.; STORER, M. S. O desenvolvimento cognitivo das crianças com síndrome de Down à luz das relações familiares. Psicologia: teoria e prática, [S.1], v. 4, n. 2, p. 31-40, 2002.

WORLD HEALTH ORGANIZATION. Obesity: preventing and managing the global epidemic. WHO Technical Report Series n. 894, 2000. 
78 Universitas: Ciências da Saúde, Brasília, v. 8, n. 1, p. 63-78, 2010

Tácio Rodrigues da S. Santos, Francisco M. da Silva, Renata Aparecida E. Dantas,

Sérgio A. Gomes, Marcelo G. B. do Nascimento, Márcio R. Mota

ZAUSMER, E. Estimulação do desenvolvimento da motricidade grossa. In: PUESCHEL, S. M. Síndrome de Down: guia para pais e educadores. Campinas: Papirus, 2007. p. 127-148. 